\title{
Twisted Alexander polynomials of periodic knots
}

\author{
JONATHAN A HiLLMAN \\ CHARLES LIVINGSTON \\ SWATEE NAIK
}

\begin{abstract}
Murasugi discovered two criteria that must be satisfied by the Alexander polynomial of a periodic knot. We generalize these to the case of twisted Alexander polynomials. Examples demonstrate the application of these new criteria, including to knots with trivial Alexander polynomial, such as the two polynomial 1 knots with 11 crossings.

Hartley found a restrictive condition satisfied by the Alexander polynomial of any freely periodic knot. We generalize this result to the twisted Alexander polynomial and illustrate the applicability of this extension in cases in which Hartley's criterion does not apply.
\end{abstract}

$57 \mathrm{M} 25,57 \mathrm{M} 27$

\section{Introduction}

The twisted Alexander polynomial of a knot, discovered independently by Jiang-Wang [21], Lin [28] and Wada [41], has seen a growing number of applications to knot theory over the last ten years. These range from the study of reversibility, mutation and concordance of knots, Kirk-Livingston [22; 23] and Tamulis [38], to identifying fibered knots, Cha [6], Goda-Morifuji [15] and Goda-Kitano-Morifuji [14]. Most recently, Friedl and Kim [13] have demonstrated that the twisted polynomial is sufficient to determine the genus and fibering properties of all prime knots of 12 crossings and less. Other literature on this invariant includes Cogolludo-Florens [8], Kitano [24], Kitano-Suzuki [25], Li-Xu [27] and Morifuji [30; 31]. In this paper we extend the application of the twisted polynomial to the study of knot periodicity.

A knot $K \subset S^{3}$ is called periodic, of period $q$, if there is an order $q$ transformation of $S^{3}$ that leaves $K$ invariant and has as fixed point set a circle $A$ disjoint from $K$. The first major result in the study of knot periodicity was the development of algebraic obstructions by Trotter [40]. The central result concerning algebraic properties of periodic knots is Murasugi's theorem [32] (see also Hillman [17] and Sakuma [36]), describing the Alexander polynomial of a periodic knot, $\Delta_{K}(t)$, in terms of $\Delta_{\bar{L}}(t, s)$, 
the Alexander polynomial of the quotient link, $\bar{L}=\bar{K} \cup \bar{A}$. From this, two conditions that must be satisfied by the Alexander polynomial of a periodic knot follow.

Theorem 1 If $K$ is periodic of period $q$ with quotient $\bar{K}$, then there is a polynomial $F(t, s) \in \mathbb{Z}\left[t^{ \pm 1}, s^{ \pm 1}\right]$ such that

$$
\Delta_{K}(t)=\Delta_{\bar{K}}(t) \prod_{i=1}^{q-1} F\left(t, \zeta_{q}^{i}\right)
$$

where $\zeta_{q}$ is a primitive $q$-root of unity.

In a more precise statement of this theorem, it is seen that $F(t, s)=\Delta_{\bar{L}}(t, s)$, and $F(t, 1)=\delta_{\lambda}(t) \Delta_{\bar{K}}(t)$ where $\delta_{\lambda}(t)=\left(1-t^{\lambda}\right) /(1-t)$ and $\lambda=\operatorname{lk}(K, A)$.

Theorem 2 If $K$ is of prime power period $q=p^{r}$ then

$$
\Delta_{K}(t) \equiv \Delta_{\bar{K}}(t)^{q} \delta_{\lambda}(t)^{q-1} \bmod p .
$$

Since the publication of Murasugi [32], our understanding of periodicity of knots has greatly expanded. In addition to a deeper understanding of Murasugi's result (Burde [4], Burde-Zieschang [5], Davis-Livingston [9; 10], Hillman [17; 18] and Sakuma [36]) new techniques have been applied, including general knot polynomials, minimal surfaces and hyperbolic geometry; examples of such work include Adams-HildebrandWeeks [1], Edmonds [12], Naik [33; 34], Przytycki [35] Traczyk [39] and Yokota [43]. However, the Murasugi conditions continue to be of central interest to the subject; in addition to being easily applied to examples, their homological nature enables their extension and application in other settings, including knots in more general manifolds and higher dimensional knots.

In classical knot theory, in the case that abelian invariants do not resolve questions of interest, the next step has been to move to nonabelian, for example dihedral, invariants of a knot. Such invariants are placed in the context of homological invariants and combined with invariants of the infinite cyclic cover of a knot via the twisted Alexander polynomial.

Later we will give a detailed description of the twisted Alexander polynomial. Briefly, given a link $L$ of $k$ components and a representation $\rho: \pi_{1}\left(S^{3}-L\right) \rightarrow G L_{n}(R)$ for some Noetherian unique factorization domain (such as a PID, ie, principal ideal domain, or field) $R$, there is a polynomial invariant

$$
\Delta(L, \rho)\left(t_{1}, \ldots, t_{k}\right) \in R\left[t_{1}^{ \pm 1}, \ldots, t_{k}^{ \pm 1}\right],
$$


well defined up to multiplication by a unit. We prove the following analogs of Murasugi's Theorems 1 and 2.

Theorem 3 Suppose that $K$ is of period $q$, and $A, \bar{K}$, and $\bar{A}$ are as above. Further, assume that $\bar{\rho}: \pi_{1}\left(S^{3}-\bar{K}\right) \rightarrow G L_{n}(R)$, with $R=\mathbb{Z}$ or $\mathbb{Q}$, and $\rho$ is the lift of that representation to $S^{3}-K$. Then there is a polynomial $F(t, s) \in R\left[t^{ \pm 1}, s^{ \pm 1}\right]$ such that

$$
\Delta_{K, \rho}(t)=\Delta_{\bar{K}, \bar{\rho}}(t) \prod_{i=1}^{q-1} F\left(t, \zeta_{q}^{i}\right) .
$$

Theorem 4 For a knot $K$ of period $q=p^{r}, p$ prime, and a representation $\bar{\rho}: \pi_{1}\left(S^{3}-\right.$ $\bar{K}) \rightarrow G L_{n}(\mathbb{Z} / p \mathbb{Z})$ with lift $\rho$, if $\Delta_{K, \rho}(t) \neq 0$, then

$$
\Delta_{K, \rho}(t)=\Delta_{\bar{K}, \bar{\rho}}(t)^{q}\left(\delta_{L, \rho}(t) / \Delta_{K, \rho}^{0}(t)\right)^{q-1} .
$$

Here $L=(K, A)$ and $\delta_{L, \rho}(t) \in(\mathbb{Z} / p \mathbb{Z})\left[t^{ \pm 1}\right]$ is more complicated than in the classical case, but will be seen in many cases to be determined by data about $K$ and $\rho$ alone. The term $\Delta^{0}$ in the denominator is related to twisted homology in dimension 0 and is easily computed in examples. In the classical setting $\Delta^{0}$ is simply $(1-t)$ and $\delta_{L, \rho}(t)=1-t^{\lambda}$, so the classical $\delta_{\lambda}$ becomes our $\delta_{L, \rho}(t) / \Delta_{K, \rho}^{0}(t)$. Also, $F$ will be seen to be related to a twisted polynomial of $\bar{L}$.

The above theorems provide obstructions to periodicity when a representation of the knot group is necessarily a lift of that for a hypothesized quotient knot. When this fails to be the case, the twisted polynomials continue to provide obstructions: there is a periodic action on the set of representations and different translates of a representation under this action must yield the same twisted polynomial.

The study of freely periodic knots, those that are invariant under a free periodic transformation of $S^{3}$, has often been treated independently of the study of periodic knots. We will see in the final section of this paper that the same techniques we use to study periodic knots apply to give results concerning the twisted Alexander polynomial of a freely periodic knot. The main result concerning Alexander polynomials of freely periodic knots was proved by Hartley in [16]. We generalize his result to the twisted case as follows, where $\bar{K}$ and $\bar{\rho}$ are defined similarly as above, though some care must be taken since the quotient space is no longer $S^{3}$.

Theorem 5 If $K$ is a freely periodic knot of period $q$ with quotient knot $\bar{K}$ having representation $\bar{\rho}$ that lifts to a representation $\rho$, then

$$
\Delta_{K, \rho}\left(t^{q}\right)=\prod_{i=0}^{q-1} \Delta_{\bar{K}, \bar{\rho}}\left(\zeta_{q}^{i} t\right) .
$$

Algebraic 83 Geometric Topology, Volume 6 (2006) 
Outline of Paper In the next section we will review the general theory of twisted homology and its use in defining the twisted Alexander modules of a knot. Section 3 considers torsion invariants of modules, gives the definition of twisted Alexander polynomials of knots and links, and describes techniques for their computation. In Section 4 we relate the twisted Alexander polynomial of a knot $K$ to that of a related link, $L=(K, A)$. Section 5 applies Shapiro's Lemma, which relates the twisted homology of a space to that of a covering space, to give splitting theorems for the twisted homology of links associated to periodic knots. The results of Section 5 are applied in Section 6 to prove Theorem 3 above, the generalization to twisted Alexander polynomials of Murasugi's result on integral polynomials, which we stated in Theorem 1. Section 7 proves Theorem 4 , the twisted analog of Murasugi's $\mathbb{Z} / p \mathbb{Z}$ result stated in Theorem 2. In Section 8 we provide basic examples illustrating these results and in Section 9 we give more delicate examples using knots with Alexander polynomial 1. Section 10 discusses methods to use the twisted polynomial to obstruct knot periodicity when Theorems 3 and 4 do not apply because there are no representations of the quotient knot that can be lifted. Note that, as all our examples are of hyperbolic knots, SnapPea, a program written by Weeks [42] that computes the isometry group for a hyperbolic knot complement provides periods of these knots. On the other hand, the twisted polynomial approach is purely algebraic and does not require a hyperbolic structure on the knot complement. The final section, Section 11, demonstrates that the techniques of this paper extend to the study of freely periodic knots, giving an extension of a classical result of Hartley [16] to the case of twisted Alexander polynomials.

Acknowledgments The authors wish to thank Jim Davis and Paul Kirk for their helpful advice. Computations of twisted polynomials for many of the examples were run on the Beowulf cluster at University of Nevada, Reno, maintained by Eric Olson; we thank Eric for his help with the computations. The first author received support from Grey College and the University of Durham, through the Grey College Mathematics Fellowship. The second author received funding from the NSF. The third author thanks Indiana University for sabbatical support.

\section{Review of twisted homology and Alexander modules}

In this section we review the basic theory of twisted homology groups.

Let $X$ be a connected finite CW complex with fundamental group $\pi=\pi_{1}(X)$, let $R$ be a commutative ring, and let $M$ be a right $R$-module. Suppose also that $\rho: \pi \rightarrow \operatorname{Aut}_{R}(M)$ is a homomorphism. Via $\rho, M$ can be viewed as a right $R[\pi]-$ module, with $m \cdot g=\rho\left(g^{-1}\right)(m)$ for $m \in M$ and $g \in \pi$. 
Let $\tilde{X}$ denote the universal cover of $X$ and let $C_{*}=C_{*}(\tilde{X})$ denote its cellular chain complex with $R$ coefficients. This can be viewed as a left $R[\pi]$-module.

Given this, one can form the $R$-chain complex $M \otimes_{R[\pi]} C_{*}$. The homology of this complex is denoted $H_{*}(X, M)$. Note that for a ring $S$, if $M$ has a left $S$-module structure which is compatible with $\rho$ (meaning $s(m \cdot g)=(\mathrm{sm}) \cdot g$ ) then $H_{*}(X, M)$ is naturally a left $S$-module.

Two basic examples the reader should consider are the case that $M=R$ with trivial $\pi$ representation, in which case $H_{*}(X, M)=H_{*}(X, R)$, and the case that $M=R[\pi]$ with standard right $\pi$ action, in which case $H_{*}(X, M)=H_{*}(\tilde{X}, R)$. Generalizing these examples, one has the following basic theorem in homological algebra, Shapiro's Lemma. See Brown [3] for a basic reference.

Theorem 6 (Shapiro's Lemma) If $M$ is a $\pi$-module and $\kappa \subset \pi$, then $H_{*}\left(X_{\kappa}, M\right)=$ $H_{*}\left(X, M \otimes_{R} R[\pi / \kappa]\right)$, where $X_{\kappa}$ is the cover of $X$ associated to the subgroup $\kappa$ and $M \otimes_{R} R[\pi / \kappa]$ is a $\pi$-module via the diagonal action.

\subsection{Examples: Link exteriors and twisted Alexander modules}

For a link $L \subset S^{3}$ the complement $X_{L}$ has the homotopy type of a CW complex with one 0-cell, $g$ 1-cells, and $(g-1) 2$-cells. This corresponds naturally to a presentation of the fundamental group of the complement, $\pi=\left\langle x_{1}, \ldots, x_{g} \mid r_{1}, \ldots, r_{g-1}\right\rangle$.

The chain complex of the universal cover of $X_{L}$ as an $R[\pi]$-module is given by

$$
0 \rightarrow R[\pi]^{g-1} \stackrel{\partial_{2}}{\rightarrow} R[\pi]^{g} \stackrel{\partial_{1}}{\rightarrow} R[\pi] \rightarrow 0
$$

The boundary map $\partial_{1}$ is given by right multiplication by the transpose of the vector $\left[1-x_{1}, \ldots, 1-x_{g}\right]$. The boundary map $\partial_{2}$ is given by right multiplication by a $(g-1) \times g$ matrix called the Fox matrix with entries the Fox derivatives, $\partial r_{i} / \partial x_{j} \in R[\pi]$.

To define a twisted Alexander polynomial of a link, one begins with a representation $\rho: \pi \rightarrow G L_{n}(R)$. Fixing any set of meridians to distinct components, $\left\{m_{1}, \cdots, m_{r}\right\}$, there is also an action of $\pi$ on $R\left[t_{1}^{ \pm 1}, \cdots, t_{r}^{ \pm 1}\right]$, where $m_{i}$ acts by multiplication by $t_{i}$. Then $R^{n} \otimes R\left[t_{1}^{ \pm 1}, \cdots, t_{r}^{ \pm 1}\right]$ is naturally a $\pi$-module, which we abbreviate $R^{n}\left[t_{i}^{ \pm 1}\right]$. (In the case of greatest interest here, $L$ has one component or $L=K \cup A$ and only a meridian of $K$ is selected, in which case we drop the subscript and work with $R\left[t^{ \pm 1}\right]$.)

The homology of $X_{L}$ with coefficients in $R^{n}\left[t_{i}^{ \pm 1}\right]$ is given by a sequence

$$
0 \rightarrow\left(R^{n}\left[t_{i}^{ \pm 1}\right]\right)^{g-1} \stackrel{\beta_{2}}{\rightarrow}\left(R^{n}\left[t_{i}^{ \pm 1}\right]\right)^{g} \stackrel{\beta_{1}}{\rightarrow} R^{n}\left[t_{i}^{ \pm 1}\right] \rightarrow 0
$$


where $\beta_{2}$ and $\beta_{1}$ are given by $n(g-1) \times n g$ and $n g \times n$ matrices with entries in $R\left[t_{i}^{ \pm 1}\right]$.

Definition 7 The homology groups of this chain complex, the twisted Alexander modules of $L$, are denoted $A_{k}(L, \rho)\left(t_{i}\right)$. These are $R\left[t_{i}^{ \pm 1}\right]$-modules. The number of variables and the corresponding meridians are identified in context and not notated.

\section{Torsion invariants, twisted polynomials, and computations}

\subsection{Torsion invariants}

In general, let $S$ be a commutative ring with unity and suppose that $M$ is a torsion $S$-module with presentation

$$
S^{m} \stackrel{A}{\rightarrow} S^{n} \rightarrow M \rightarrow 0
$$

where $A$ is an $m \times n$ matrix with entries in $S, m \geq n$. The ideal generated by all $n \times n$ minors of $A$ is an invariant of $M$, denoted $E_{0}(M)$. (Note that $A$ is not required to be injective.) Assuming that $S$ is a Noetherian UFD (for instance, when $S$ is a polynomial ring over $\mathbb{Z}, \mathbb{Q}$, or $\mathbb{Z} / p \mathbb{Z}$ ), this ideal is contained in a unique minimal principal ideal called the order of the ideal, with generator denoted $\operatorname{Ord}(M)$. If $M$ is not torsion, we set $\operatorname{Ord}(M)=0$. There is the following result; see Theorem 3.12 of Hillman [19] for details.

Theorem 8 If $0 \rightarrow M \rightarrow N \rightarrow P \rightarrow 0$ is a short exact sequence of $S$-torsion modules, then $\operatorname{Ord}(M) \operatorname{Ord}(P)=\operatorname{Ord}(N)$.

\subsection{Twisted Alexander polynomials}

In the case of greatest interest to us, the twisted Alexander modules of a link, we have:

Definition $9 \Delta_{L, \rho}^{k}\left(t_{i}\right)=\operatorname{Ord}\left(A_{k}(L, \rho)\left(t_{i}\right)\right)$. In the case that $k=1$ we drop the superscript, $\Delta_{L, \rho}\left(t_{i}\right)=\Delta_{L, \rho}^{1}\left(t_{i}\right)$.

\subsection{Computations}

In general we are not given a presentation of $A_{k}(L, \rho)\left(t_{i}\right)$, but rather the chain complex that collectively determines these modules. Furthermore, the matrices are quite large and the number of minors that arise is larger still. Computations are simplified by the following observations, basically Lemma 4.2 of Kirk-Livingston [22]. 
Lemma 10 Suppose that one has a chain complex $\mathcal{C}$ of abelian groups:

$$
0 \rightarrow A \stackrel{a \oplus b}{\rightarrow} B \oplus C \stackrel{c+d}{\rightarrow} D \rightarrow 0
$$

with $c$ injective. Then there is a naturally defined exact sequence:

$$
0 \rightarrow H_{1}(\mathcal{C}) \rightarrow \operatorname{coker}(b) \rightarrow \operatorname{coker}(c) \rightarrow H_{0}(\mathcal{C}) \rightarrow 0
$$

Proof We offer here the following simplification of the argument in [22] which was presented only in the case that $R$ is a field.

Consider the exact sequence of chain complexes $0 \rightarrow \mathcal{C}^{\prime} \rightarrow \mathcal{C} \rightarrow \mathcal{C}^{\prime \prime} \rightarrow 0$ with the complexes corresponding to the columns of the following commutative diagram, with all the maps coming from $a, b, c, d$, and the natural inclusions and projections.

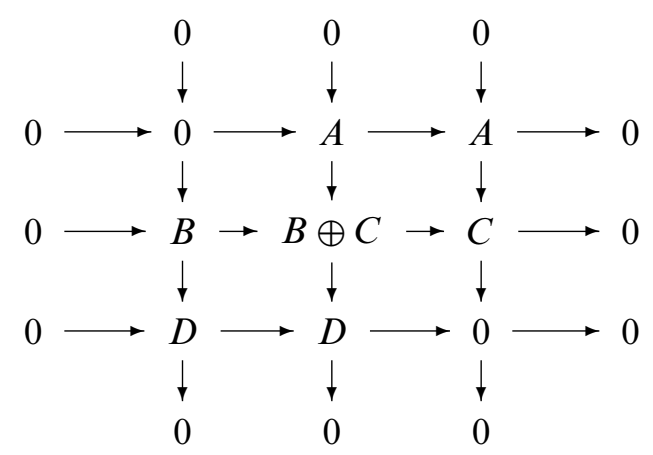

The lemma now follows from the associated long exact sequence.

We apply this in the case of the sequence given in (2-1), rewritten as

$$
0 \rightarrow\left(R\left[t_{i}^{ \pm 1}\right]\right)^{n(g-1)} \stackrel{a \oplus b}{\rightarrow}\left(R\left[t_{i}^{ \pm 1}\right]\right)^{n} \oplus\left(R\left[t_{i}^{ \pm 1}\right]\right)^{n(g-1)} \stackrel{c+d}{\rightarrow} R\left[t_{i}^{ \pm 1}\right]^{n} \rightarrow 0,
$$

where the choice of splitting of the middle group depends on a choice of an initial generator of $\pi$.

Applying the previous lemma and recognizing that the orders of the cokernels are given by determinants, we have

Theorem 11 If $A_{1}(L, \rho)$ and $A_{0}(L, \rho)$ are torsion, then

$$
\Delta_{L, \rho}\left(t_{i}\right) / \Delta_{L, \rho}^{0}\left(t_{i}\right)=\operatorname{det}(b) / \operatorname{det}(c) .
$$


In Wada [41] this quotient of determinants is defined to be an invariant of a link, now called the Wada invariant $W$, and is explicitly shown to be independent of the choice of generator of $\pi$ selected as the initial generator, and more generally, to be independent of the choice of presentation. The homological interpretation given here comes from [22], and offers an alternative proof of this independence.

\section{Relating twisted knot and link polynomials}

In our application to periodic knots, one starts with a possibly periodic knot $K$ and hypothesizes an axis $A$ for the action. One is thus forced to consider the relationship between the twisted invariants of $K$ and those of the hypothesized link $L=K \cup A$. Specifically, for the knot $K$ there is a representation $\rho: \pi \rightarrow G L_{n}(R)$ and the twisted invariants, $A_{k}(K, \rho)(t)$ and $\Delta_{K, \rho}^{k}(t)$. There is the associated link $L$ and the induced representation which we continue to denote by $\rho$. There is also an induced action of $\pi_{1}\left(X_{L}\right)$ on $R^{n}\left[t^{ \pm 1}\right]$, with the meridian of $A$ acting trivially, and thus there are twisted invariants $A_{k}(L, \rho)(t)$ and $\Delta_{L, \rho}^{k}(t)$.

Before relating these twisted invariants of $K$ and $L$, we need a bit of notation. From the chain complex we have that

$$
A_{0}(K, \rho)=R^{n}\left[t^{ \pm 1}\right] /\left\langle\left\{\left(I-\rho^{*}(g)\right) m \mid g \in \pi, m \in R^{n}\left[t^{ \pm 1}\right]\right\}\right\rangle,
$$

where $\rho^{*}(g)=t^{\epsilon(g)} \rho(g)$ and $\epsilon$ is the abelianization map $\pi \rightarrow \mathbb{Z}$. For notational simplicity, we abbreviate this quotient as $R^{n}\left[t^{ \pm 1}\right] / \operatorname{Im}\left(I-\rho^{*}\right)$. Also, we use the following:

Definition $12 \delta_{L, \rho}(t)=\operatorname{det}\left(I-\rho(A) t^{\lambda}\right)$, where $\lambda=\operatorname{lk}(K, A)$ denotes the linking number.

Lemma $13 H_{1}\left(A, R^{n}\left[t^{ \pm 1}\right]\right)=0$ and $H_{0}\left(A, R^{n}\left[t^{ \pm 1}\right]\right)$ is presented by $I-\rho(A) t^{\lambda}$ and thus has order $\delta_{L, \rho}(t)$.

Proof Since $A \cong S^{1}$, it has one $0-$ cell and one $1-$ cell. The action of $\pi_{1}(A)$ on $R^{n}\left[t^{ \pm 1}\right]$ is generated by $\rho(A) t^{\lambda}$, and thus the chain complex used to compute its homology is

$$
0 \rightarrow R^{n}\left[t^{ \pm 1}\right] \stackrel{I-\rho(A) t^{\lambda}}{\longrightarrow} R^{n}\left[t^{ \pm 1}\right] \rightarrow 0
$$

As we note at the end of the proof of the next result, $\lambda \neq 0$, so $\operatorname{det}\left(I-\rho(A) t^{\lambda}\right)$ has constant term 1 (set $t=0$ ), and in particular is nonzero. Thus the map $I-\rho(A) t^{\lambda}$ is injective and the result follows. 
Theorem 14 In the situation described above:

(a) $A_{0}(L, \rho)=A_{0}(K, \rho)=R^{n}\left[t^{ \pm 1}\right] / \operatorname{Im}\left(I-\rho^{*}\right)$, and $\Delta_{K, \rho}^{0}(t)=\Delta_{L, \rho}^{0}(t)$.

(b) If $A_{2}(K, \rho)=0$, then there is an exact sequence

$$
\begin{aligned}
& 0 \rightarrow R^{n}\left[t^{ \pm 1}\right] /\left\langle\left(I-\rho(A) t^{\lambda}\right)(m) \mid m \in R^{n}\left[t^{ \pm 1}\right]\right\rangle \rightarrow A_{1}(L, \rho) \rightarrow A_{1}(K, \rho) \rightarrow 0, \\
& \text { where } \lambda=1 k(K, A) . \\
& \text { (c) If } A_{2}(K, \rho)=0 \text {, then } \Delta_{L, \rho}(t)=\delta_{L, \rho}(t) \Delta_{K, \rho}(t) . \\
& \text { (d) If } A_{2}(K, \rho)=0 \text {, then } A_{2}(L, \rho)=0 .
\end{aligned}
$$

Proof Statement (a) follows from the definition of the twisted homology groups and that the image of $\rho^{*}$ is the same, whether applied to $\pi_{1}\left(X_{K}\right)$ or $\pi_{1}\left(X_{L}\right)$.

For part (b), the Mayer-Vietoris sequence associated to the decomposition $X_{K}=$ $X_{L} \cup N(A)$ yields the following, in which the twisted coefficients have been left out of the notation.

$$
\begin{aligned}
0 \rightarrow H_{1}\left(T^{2}\right) & \rightarrow H_{1}(A) \oplus H_{1}\left(X_{L}\right) \rightarrow H_{1}\left(X_{K}\right) \rightarrow \\
& H_{0}\left(T^{2}\right) \rightarrow H_{0}(A) \oplus H_{0}\left(X_{L}\right) \rightarrow H_{0}\left(X_{K}\right) \rightarrow 0 .
\end{aligned}
$$

Since the representation is trivial on the meridian of $A$, the map $H_{0}\left(T^{2}\right) \rightarrow H_{0}(A)$ is an isomorphism. This, along with the explicit calculation that $H_{1}\left(T^{2}\right)=R^{n}\left[t^{ \pm 1}\right] /$ $\left\langle\left(I-\rho(A) t^{\lambda}\right)(m) \mid m \in R^{n}\left[t^{ \pm 1}\right]\right\rangle$ and that $H_{1}(A)=0$, yields the next sequence.

$$
0 \rightarrow R^{n}\left[t^{ \pm 1}\right] /\left\langle I-\rho(A) t^{\lambda}\right\rangle \rightarrow H_{1}\left(X_{L}\right) \rightarrow H_{1}\left(X_{K}\right) \rightarrow 0 .
$$

Part (c) follows from (b).

For part (d), from the Mayer-Vietoris sequence

$$
0 \rightarrow H_{2}\left(T^{2}\right) \rightarrow H_{2}(A) \oplus H_{2}\left(X_{L}\right) \rightarrow H_{2}\left(X_{K}\right)=0,
$$

we conclude, since $H_{2}(A)=0$ ( $A$ is a 1-complex), $H_{2}\left(X_{L}\right)=H_{2}\left(T^{2}\right)$. From an explicit calculation we have $H_{2}\left(T^{2}\right)=\operatorname{ker}\left(I-\rho(A) t^{\lambda}\right)$. But clearly the determinant of $I-\rho(A) t^{\lambda}$ is a nonzero polynomial: as we recall in the next paragraph, $\operatorname{gcd}(\lambda, q)=1$, so $\lambda \neq 0$ and the constant term in $\operatorname{det}\left(I-\rho(A) t^{\lambda}\right)$ is 1 .

To conclude, we need to explain why $\operatorname{gcd}(\lambda, q)=1$. The knot $K$ is the preimage of $\bar{K}$ in the $q$-fold cyclic cover of $S^{3}-\bar{A}$. Since $\bar{K}$ is connected, the number of components of $K, 1$, is given by $\operatorname{gcd}(q, \operatorname{lk}(\bar{K}, \bar{A}))$. The cyclic cover can be constructed using the Seifert surface for $\bar{A}$, and $\operatorname{lk}(\bar{K}, \bar{A})$ is the algebraic intersection of $\bar{K}$ with that surface. Similarly, $\lambda=\operatorname{lk}(K, A)$ is computed as the algebraic intersection of $K$ with a lift of the Seifert surface, which is clearly the same number. 


\section{Application of Shapiro's Lemma to periodic knots}

In this section we work with a field $R$, which is either $\mathbb{Q}\left[\zeta_{q}\right]$ or $\mathbb{Z} / p \mathbb{Z}$, where $\zeta_{q}$ is a fixed primitive $q$-root of unity and $p$ is prime.

Suppose that $K$ is invariant under a $\mathbb{Z} / q \mathbb{Z}$ action on $S^{3}$ with fixed point set a circle $A$ disjoint from $K$. We can form the quotient link $\bar{L}=(\bar{K}, \bar{A})$. Recall that $\rho: \pi_{1}\left(X_{K}\right) \rightarrow$ $G L_{n}(R)$ and that $R^{n}\left[t^{ \pm 1}\right] \cong R^{n} \otimes R\left[t^{ \pm 1}\right]$ is viewed as a $\pi_{1}\left(X_{K}\right)$-module. In addition, assume that there is a representation $\bar{\rho}: \pi_{1}\left(X_{\bar{K}}\right) \rightarrow G L_{n}(R)$ of which our original $\rho$ is the lift. Shapiro's Lemma yields the following.

Theorem $15 H_{1}\left(X_{L}, R^{n}\left[t^{ \pm 1}\right]\right)=H_{1}\left(X_{\bar{L}}, R^{n}\left[t^{ \pm 1}\right] \otimes R[\mathbb{Z} / q \mathbb{Z}]\right)$.

Here the module $R^{n}\left[t^{ \pm 1}\right] \otimes R[\mathbb{Z} / q \mathbb{Z}]$ is acted on diagonally by $\pi_{1}\left(X_{\bar{L}}\right)$, the meridian of $\bar{K}$ acting trivially on $R[\mathbb{Z} / q \mathbb{Z}]$ and the meridian of $\bar{A}$ acting by multiplication by a fixed generator of $\mathbb{Z} / q \mathbb{Z}$ on $R[\mathbb{Z} / q \mathbb{Z}]$.

\subsection{Application 1: $\quad R=\mathbb{Q}\left[\zeta_{q}\right]$}

In this case there is a splitting of the $\mathbb{Z} / q \mathbb{Z}$-module: $R[\mathbb{Z} / q \mathbb{Z}]=\oplus_{i=0}^{q-1} \mathbb{Q}\left[\zeta_{q}^{i}\right]$, where $\mathbb{Q}\left[\zeta_{q}^{i}\right]$ is viewed as an $R[\mathbb{Z} / q \mathbb{Z}]$-module, with the generator of $\mathbb{Z} / q \mathbb{Z}$ acting on $\mathbb{Q}\left[\zeta_{q}^{i}\right]$ by multiplication by $\zeta_{q}^{i}$. Hence, we have the next result.

Theorem $16 H_{1}\left(X_{L}, R^{n}\left[t^{ \pm 1}\right]\right)=\oplus_{i=0}^{q-1} H_{1}\left(X_{\bar{L}}, R^{n}\left[t^{ \pm 1}\right] \otimes \mathbb{Q}\left[\zeta_{q}^{i}\right]\right)$.

Remark Note that $R^{n}\left[t^{ \pm 1}\right] \otimes \mathbb{Q}\left[\zeta_{q}^{i}\right] \cong R^{n}\left[t^{ \pm 1}\right]$ with the $\mathbb{Z} / q \mathbb{Z}$ action given by multiplication by $\zeta_{q}^{i}$. In particular, $H_{1}\left(X_{\bar{L}}, R^{n}\left[t^{ \pm 1}\right] \otimes \mathbb{Q}\left[\zeta_{q}^{0}\right]\right)=H_{1}\left(X_{\bar{L}}, R^{n}\left[t^{ \pm 1}\right]\right)$ with trivial $\mathbb{Z} / q \mathbb{Z}$ action.

\subsection{Application 2: $\quad R=\mathbb{Z} / p \mathbb{Z}$ and $q=p^{r}$ for some prime $p$.}

In this case, $R[\mathbb{Z} / q \mathbb{Z}]=(\mathbb{Z} / p \mathbb{Z})[g] /\left\langle 1-g^{q}\right\rangle$, where $(\mathbb{Z} / p \mathbb{Z})[g]$ is the polynomial ring in the variable $g$. Since the coefficients are in $\mathbb{Z} / p \mathbb{Z}$, this equals $(\mathbb{Z} / p \mathbb{Z})[g] /\left\langle(1-g)^{q}\right\rangle$. In general, write $(\mathbb{Z} / p \mathbb{Z})[g] /\left\langle(1-g)^{k}\right\rangle=V_{k}$ and note that $V_{1}=\mathbb{Z} / p \mathbb{Z}$. The following sequence of modules is exact:

$$
0 \rightarrow V_{k-1} \rightarrow V_{k} \rightarrow V_{1} \rightarrow 0,
$$

where the map $V_{k-1} \rightarrow V_{k}$ is given by multiplication by $1-g$. Theorem 15 gives that $H_{1}\left(X_{L}, R^{n}\left[t^{ \pm 1}\right]\right)=H_{1}\left(X_{\bar{L}}, R^{n}\left[t^{ \pm 1}\right] \otimes V_{q}\right)$. We now have the following result, a "splitting theorem" for the twisted homology, that will provide the basis for an inductive argument computing the twisted Alexander polynomial for $\mathbb{Z} / p \mathbb{Z}$-coefficients in Section 7. 
Theorem 17 If $H_{2}\left(X_{K}, R^{n}\left[t^{ \pm 1}\right]\right)=0$, then for $k>1$ there is the following exact sequence:

$$
\begin{aligned}
0 \rightarrow H_{1}\left(X_{\bar{L}}, R^{n}\left[t^{ \pm 1}\right] \otimes V_{k-1}\right) \rightarrow H_{1}\left(X_{\bar{L}}, R^{n}\left[t^{ \pm 1}\right] \otimes V_{k}\right) \rightarrow \\
H_{1}\left(X_{\bar{L}}, R^{n}\left[t^{ \pm 1}\right]\right) \rightarrow H_{0}\left(X_{K}, R^{n}\left[t^{ \pm 1}\right]\right) \rightarrow 0 .
\end{aligned}
$$

Proof Note first that $H_{1}\left(X_{\bar{L}}, R^{n}\left[t^{ \pm 1}\right] \otimes V_{1}\right)=H_{1}\left(X_{\bar{L}}, R^{n}\left[t^{ \pm 1}\right]\right)$, since $V_{1}=\mathbb{Z} / p \mathbb{Z}$.

The tensor products are taken over $\mathbb{Z} / p \mathbb{Z}$, and $R^{n}\left[t^{ \pm 1}\right]$ is free as a $\mathbb{Z} / p \mathbb{Z}$ vector space, so tensoring with it preserves exactness and the following sequence is exact:

$$
0 \rightarrow R^{n}\left[t^{ \pm 1}\right] \otimes V_{k-1} \rightarrow R^{n}\left[t^{ \pm 1}\right] \otimes V_{k} \rightarrow R^{n}\left[t^{ \pm 1}\right] \otimes V_{1} \rightarrow 0 .
$$

Observation $1 \quad H_{2}\left(X_{\bar{L}}, R^{n}\left[t^{ \pm 1}\right] \otimes V_{1}\right)=0$.

Since $H_{2}\left(X_{K}, R^{n}\left[t^{ \pm 1}\right]\right)=0$, by Theorem 14 we have that $H_{2}\left(X_{L}, R^{n}\left[t^{ \pm 1}\right]\right)=0$. By Shapiro's Lemma, we conclude that $H_{2}\left(X_{\bar{L}}, R^{n}\left[t^{ \pm 1}\right] \otimes(\mathbb{Z} / p \mathbb{Z})[g] /\left\langle 1-g^{q}\right\rangle\right)=0$. Since the coefficients are in $\mathbb{Z} / p \mathbb{Z}$ this is the same as $H_{2}\left(X_{\bar{L}}, R^{n}\left[t^{ \pm 1}\right] \otimes V_{q}\right)=0$.

Consider now the exact sequence coming from the exact sequence of coefficients:

$$
H_{3}\left(X_{\bar{L}}, R^{n}\left[t^{ \pm 1}\right] \otimes V_{1}\right) \rightarrow H_{2}\left(X_{\bar{L}}, R^{n}\left[t^{ \pm 1}\right] \otimes V_{k-1}\right) \rightarrow H_{2}\left(X_{\bar{L}}, R^{n}\left[t^{ \pm 1}\right] \otimes V_{k}\right) .
$$

Since we are working with homotopy 2-complexes, the first term is 0 and thus for all $k, H_{2}\left(X_{\bar{L}}, R^{n}\left[t^{ \pm 1}\right] \otimes V_{k-1}\right) \rightarrow H_{2}\left(X_{\bar{L}}, R^{n}\left[t^{ \pm 1}\right] \otimes V_{k}\right)$ is injective. Starting with the fact that $H_{2}\left(X_{\bar{L}}, R^{n}\left[t^{ \pm 1}\right] \otimes V_{q}\right)=0$ and working down, we conclude that $H_{2}\left(X_{\bar{L}}, R^{n}\left[t^{ \pm 1}\right] \otimes V_{1}\right)=0$, as desired.

Observation $2 H_{0}\left(X_{\bar{L}}, R^{n}\left[t^{ \pm 1}\right] \otimes V_{k-1}\right) \rightarrow H_{0}\left(X_{\bar{L}}, R^{n}\left[t^{ \pm 1}\right] \otimes V_{k}\right)$ is the zero map.

In general, $H_{0}\left(X_{\bar{L}}, R^{n}\left[t^{ \pm 1}\right] \otimes V_{k}\right)$ is given as the quotient of $R^{n}\left[t^{ \pm 1}\right] \otimes V_{k}$ by the ideal generated by all elements of the form $(I-\bar{\rho})(\alpha)(x \otimes 1)$, where $\alpha \in \pi_{1}\left(X_{\bar{L}}\right)$, $x \in R^{n}\left[t^{ \pm 1}\right]$, and $\bar{\rho}: \pi_{1}\left(X_{\bar{L}}\right) \rightarrow \operatorname{Aut}\left(R^{n}\left[t^{ \pm 1}\right] \otimes V_{k}\right)$, the action defined earlier on $R^{n}\left[t^{ \pm 1}\right]$ extended as described to $R^{n}\left[t^{ \pm 1}\right] \otimes V_{k}$.

A meridian to $\bar{A}$ acts trivially on $R^{n}\left[t^{ \pm 1}\right]$ and acts by multiplication by $g$ on $V_{k}$. Thus, in the quotient space, $1-g$ acts trivially. On the other hand, the map from $V_{k-1}$ to $V_{k}$ is given by multiplication by $1-g$, and hence the induced map on homology is trivial.

Observation 3 There exists a natural isomorphism,

$$
H_{0}\left(X_{\bar{L}}, R^{n}\left[t^{ \pm 1}\right] \otimes V_{k}\right) \rightarrow H_{0}\left(X_{K}, R^{n}\left[t^{ \pm 1}\right]\right) .
$$


Since $V_{k} /\langle 1-g\rangle=\mathbb{Z} / p \mathbb{Z}$, the inclusion $H_{0}\left(X_{\bar{L}}, R^{n}\left[t^{ \pm 1}\right]\right) \rightarrow H_{0}\left(X_{\bar{L}}, R^{n}\left[t^{ \pm 1}\right] \otimes V_{k}\right)$ is an isomorphism. The inclusion $X_{\bar{L}} \rightarrow X_{\bar{K}}$ is surjective on $\pi_{1}$, so induces an isomorphism on $H_{0}$ with twisted coefficients. Similarly, the projection $X_{K} \rightarrow X_{\bar{K}}$ is surjective on $\pi_{1}$, so this too induces an isomorphism on $H_{0}$ with twisted coefficients.

\section{Murasugi's condition for twisted polynomials of periodic knots with integral or rational representations}

Here we prove our main result concerning integral and rational twisted polynomials of periodic knots:

Theorem 3 Suppose that $K$ is of period $q$, and $A, \bar{K}$, and $\bar{A}$ are as above. Further, assume that $\bar{\rho}: \pi_{1}\left(S^{3}-\bar{K}\right) \rightarrow G L_{n}(R)$, with $R=\mathbb{Z}$ or $\mathbb{Q}$, and $\rho$ is the lift of that representation to $S^{3}-K$. If $\Delta_{K, \rho}(t) \neq 0$, then there is a polynomial $F(t, s) \in$ $R\left[t^{ \pm 1}, s^{ \pm 1}\right]$ such that

$$
\Delta_{K, \rho}(t)=\Delta_{\bar{K}, \bar{\rho}}(t) \prod_{i=1}^{q-1} F\left(t, \zeta_{q}^{i}\right)
$$

Note that since $\Delta_{K, \rho}(t) \neq 0, A_{2}(K, \rho)=0$ and Theorem 14 applies.

Let $R=\mathbb{Z}$ or $\mathbb{Q}$. The Alexander polynomial of interest is associated to the homology group $H_{1}\left(X_{K}, R^{n}\left[t^{ \pm 1}\right]\right.$ ). We can tensor $R^{n}$ with $\mathbb{Q}\left[\zeta_{q}\right]$ to form the module $R_{\zeta}^{n}$. (Notice that $R_{\zeta}=\mathbb{Q}\left[\zeta_{q}\right]$, but writing $R_{\zeta}$ will reduce confusion.) Denote the representation obtained by tensoring as $\rho_{\zeta}$. Observe that $\Delta_{K, \rho}(t)=\Delta_{K, \rho_{\zeta}}(t)$, as follows from the computational formula provided by Theorem 11.

By Theorem 14 (part c), $\Delta_{K, \rho_{\zeta}}(t) \delta_{L, \rho}(t)=\Delta_{L, \rho_{\zeta}}(t)$. Next, we apply Theorem 16:

$$
\Delta_{L, \rho_{\zeta}}(t)=\Delta_{\bar{L}, \bar{\rho}_{\zeta}}(t) \prod_{i=1}^{q-1} \operatorname{Ord} H_{1}\left(X_{\bar{L}}, R^{n}\left[t^{ \pm 1}\right] \otimes \mathbb{Q}\left[\zeta_{q}^{i}\right]\right) .
$$

Again by Theorem 14 (part c), $\Delta_{\bar{L}, \bar{\rho}_{\zeta}}(t)=\Delta_{\bar{K}, \bar{\rho}_{\zeta}}(t) \delta_{\bar{L}_{\bar{L}} \bar{\rho}_{\zeta}}(t)$. But note that $\delta_{\bar{L}, \bar{\rho}_{\zeta}}(t)=$ $\delta_{L, \rho}(t)$, since $\lambda$ is the same for $L$ and $\bar{L}$ as is $\rho(A)$ and $\overline{\rho_{\zeta}}(\bar{A})$.

Then, canceling the $\delta_{\lambda}$ terms, we have:

$$
\Delta_{K, \rho_{\zeta}}(t)=\Delta_{\bar{K}, \bar{\rho}_{\zeta}}(t) \prod_{i=1}^{q-1} \operatorname{Ord} H_{1}\left(X_{\bar{L}}, R_{\zeta}^{n} \otimes \mathbb{Q}\left[\zeta_{q}^{i}\right]\right) .
$$

Algebraic $8 \mathcal{G}$ Geometric Topology, Volume 6 (2006) 
Thus, it remains to analyze $\operatorname{Ord} H_{*}\left(X_{\bar{L}}, R_{\zeta}^{n}\left[t^{ \pm 1}\right] \otimes \mathbb{Q}\left[\zeta_{q}^{i}\right]\right)$. Suppose that $\pi_{1}\left(S^{3}-\bar{L}\right)$ has a presentation with $g$ generators and $g-1$ relations, and assume further that the first of the generators, $x_{1}$, is a meridian to the axis. There is an action of $\pi_{1}\left(S^{3}-\bar{L}\right)$ on $R^{n}\left[t^{ \pm 1}\right] \otimes \mathbb{Q}\left[s^{ \pm 1}\right]=R^{n}\left[t^{ \pm 1}, s^{ \pm 1}\right]$, where the meridians to $\bar{A}$ act on $\mathbb{Q}[s]$ by multiplication by $s$, and meridians to $\bar{K}$ act on $\mathbb{Q}[s]$ by multiplication by 1 . The homology groups of $X_{\bar{L}}$ with these coefficients, the two variable twisted Alexander modules of $\bar{L}$, are determined by an exact sequence:

$$
\begin{array}{r}
0 \rightarrow\left(R^{n}\left[t^{ \pm 1}, s^{ \pm 1}\right]\right)^{g-1} \stackrel{A(s, t) \oplus B(s, t)}{\longrightarrow}\left(R^{n}\left[t^{ \pm 1}, s^{ \pm 1}\right]\right) \oplus\left(R^{n}\left[t^{ \pm 1}, s^{ \pm 1}\right]\right) \\
\stackrel{C(t, s)+D(t, s)}{\longrightarrow} R^{n}\left[t^{ \pm 1}, s^{ \pm 1}\right] \rightarrow 0 .
\end{array}
$$

Replacing $s$ with $\zeta_{q}^{i}$ in this sequence (that is, tensoring over $\mathbb{Q}[s]$ with $\mathbb{Q}\left[\zeta_{q}^{i}\right]$ with $s$ acting on $\mathbb{Q}\left[\zeta_{q}^{i}\right]$ by multiplication by $\zeta_{q}^{i}$ ) gives the sequence used to compute the homology $H_{*}\left(X_{\bar{L}}, R_{\zeta}^{n}\left[t^{ \pm} 1\right] \otimes \mathbb{Q}\left[\zeta_{q}^{i}\right]\right)$.

The determinant of $C(t, s)$ is simply $(1-s)^{n}$, and this is nonzero if $s=\zeta_{q}^{i}, 1 \leq i<q$. Hence, by Theorem 11,

$$
\begin{aligned}
& \operatorname{Ord} H_{1}\left(X_{\bar{L}}, R_{\zeta}^{n}\left[t^{ \pm 1}\right] \otimes \mathbb{Q}\left[\zeta_{q}^{i}\right]\right)= \\
& \quad \operatorname{det}\left(B\left(t, \zeta_{q}^{i}\right)\right) \cdot \operatorname{Ord} H_{0}\left(X_{\bar{L}}, R^{n}\left[t^{ \pm 1}\right] \otimes \mathbb{Q}\left[\zeta_{q}^{i}\right]\right) / \operatorname{det}\left(C\left(t, \zeta_{q}^{i}\right)\right) .
\end{aligned}
$$

However, since $C\left(t, \zeta_{q}^{i}\right)=\left(1-\zeta_{q}^{i}\right) I$ and $1-\zeta_{q}^{i}$ is nonzero, the homology group $H_{0}\left(X_{\bar{L}}, R^{n}\left[t^{ \pm 1}\right] \otimes \mathbb{Q}\left[\zeta_{q}^{i}\right]\right)=0$. Letting $G(t, s)=\operatorname{det}(B(t, s)) /(1-s)^{n}$, we now have:

Theorem 18 In the above situation,

$$
\Delta_{K, \rho_{\zeta}}(t)=\Delta_{\bar{K}, \bar{\rho}_{\zeta}}(t) \prod_{i=1}^{q-1} G\left(t, \zeta_{q}^{i}\right) .
$$

It is not clear initially that $G(t, s)$ is a polynomial, because of the factor $(1-s)^{n}$ in the denominator. However we do have the following fact (see also [41]).

Lemma $19 G(t, s) \in R\left[t^{ \pm 1}, s^{ \pm 1}\right]$.

Proof If we had made a meridian to $\bar{K}$, say $m$, the first generator instead of the meridian to $\bar{A}$, we would have had a similar sequence to $(6-1)$ :

$$
\begin{aligned}
0 \rightarrow\left(R^{n}\left[t^{ \pm 1}, s^{ \pm 1}\right]\right)^{g-1} \stackrel{A^{*}(s, t) \oplus B^{*}(s, t)}{\longrightarrow}\left(R^{n}\left[t^{ \pm 1}, s^{ \pm 1}\right]\right) \oplus\left(R^{n}\left[t^{ \pm 1}, s^{ \pm 1}\right]\right) & (g-1) \\
& \stackrel{C^{*}(t, s)+D^{*}(t, s)}{\longrightarrow} R^{n}\left[t^{ \pm 1}, s^{ \pm 1}\right] \rightarrow 0 .
\end{aligned}
$$


Now we can define $G^{*}(t, s)=\operatorname{det}\left(B^{*}((t, s)) / \operatorname{det}(I-\bar{\rho}(m) t)\right.$. According to Theorem 11 , the choice of initial generator does not affect the quotient, and thus $G(t, s)=$ $G^{*}(t, s)$. Written otherwise,

$$
\operatorname{det}(B(t, s)) \operatorname{det}(I-\bar{\rho}(m) t)=\operatorname{det}\left(B^{*}(t, s)\right)(1-s)^{n} .
$$

Since $\mathbb{Q}\left[\zeta_{q}\right]\left[t^{ \pm 1}, s^{ \pm 1}\right]$ is a UFD, $\operatorname{det}(B(t, s))$ is divisible by $(1-s)^{n}$ and the result follows.

\section{Murasugi's condition for twisted polynomials of periodic knots with $\mathbb{Z} / p \mathbb{Z}$ representations}

Here we prove our main theorem regarding twisted polynomials with $\mathbb{Z} / p \mathbb{Z}$-coefficients of periodic knots.

Theorem 4 For a knot $K$ of period $q=p^{r}, p$ prime, and a representation $\bar{\rho}: \pi_{1}\left(S^{3}-\right.$ $\bar{K}) \rightarrow G L_{n}(\mathbb{Z} / p \mathbb{Z})$ with lift $\rho$, if $\Delta_{K, \rho}(t) \neq 0$, then

$$
\Delta_{K, \rho}(t)=\Delta_{\bar{K}, \bar{\rho}}(t)^{q}\left(\delta_{L, \rho}(t) / \Delta_{K, \rho}^{0}(t)\right)^{q-1} .
$$

By Theorem 17, we have that

$$
\begin{aligned}
\operatorname{Ord} H_{1}\left(X_{\bar{L}}, R^{n}\left[t^{ \pm 1}\right] \otimes V_{q}\right) & \operatorname{Ord} H_{0}\left(X_{K}, R^{n}\left[t^{ \pm 1}\right]\right)= \\
& \operatorname{Ord} H_{1}\left(X_{\bar{L}}, R^{n}\left[t^{ \pm 1}\right] \otimes V_{q-1}\right) \operatorname{Ord} H_{1}\left(X_{\bar{L}}, R^{n}\left[t^{ \pm 1}\right]\right) .
\end{aligned}
$$

Dividing by the $H_{0}$ term and proceeding by induction to the case of $V_{q-(q-1)}=\mathbb{Z} / p \mathbb{Z}$, we find that

$$
\begin{aligned}
& \operatorname{Ord} H_{1}\left(X_{\bar{L}}, R^{n}\left[t^{ \pm 1}\right] \otimes V_{q}\right)= \\
& \quad \operatorname{Ord} H_{1}\left(X_{\bar{L}}, R^{n}\left[t^{ \pm 1}\right]\right)\left(\operatorname{Ord} H_{1}\left(X_{\bar{L}}, R^{n}\left[t^{ \pm 1}\right]\right) / \operatorname{Ord} H_{0}\left(X_{K}, R^{n}\left[t^{ \pm 1}\right]\right)\right)^{q-1} .
\end{aligned}
$$

Applying Shapiro's Lemma, Theorem 15, and switching notation, this gives that

$$
\Delta_{L, \rho}(t)=\Delta_{\bar{L}, \bar{\rho}}(t)^{q} / \Delta_{K, \rho}^{0}(t)^{q-1} .
$$

Since $\Delta_{K, \rho}(t) \neq 0$ we have $A_{2}(K, \rho)=0$. We then have, by Theorem 14, that $\Delta_{K, \rho}(t) \delta_{L, \rho}(t)=\Delta_{L, \rho}(t)$, and similarly for $\bar{K}$ and $\bar{L}$, with $\delta$ defined as before. Thus,

$$
\Delta_{K, \rho}(t) \delta_{L, \rho}(t)=\left(\Delta_{\bar{K}, \bar{\rho}}(t) \delta_{\bar{L}, \bar{\rho}}(t)\right)^{q} / \Delta_{K, \rho}^{0}(t)^{q-1} .
$$

As we have observed earlier, $\delta_{L}=\delta_{\bar{L}}$, so further simplification completes the proof of the theorem. 


\section{Examples}

We use the classical numbering system for prime knots of 10 or fewer crossings, eliminating the duplicate $10_{161}=10_{162}$ that appeared in early tables. There are 165 prime knots of 10 crossings. For prime knots of 11 and 12 crossings we use the numbering system based on Dowker-Thistlethwaite notation [11]. This number system is used by the program Knotscape, developed by Hoste and Thistlethwaite, [20]. An up-to-date table of knots through 12 crossings is available on-line at [29].

Consider the knot $10_{162}$, having irreducible Alexander polynomial

$$
\Delta_{10_{162}}(t)=3 t^{4}-9 t^{3}+11 t^{2}-9 t+3 .
$$

We first observe that Murasugi's conditions do not obstruct this knot from having period 3. One simply checks that letting $\Delta_{\bar{K}}(t)=\Delta_{10_{162}}(t)$ and $\lambda=1$ yields a solution to the mod 3 condition of Theorem 2 .

Now, for the $\mathbb{Z}\left[\zeta_{3}\right]$ condition in Theorem 1, one lets $\alpha=1+s+s^{2}$ and

$$
F(t, s)=\alpha t^{4}-3 \alpha t^{3}+(4 \alpha-1) t^{2}-3 \alpha t+\alpha .
$$

We want to see a bit more, that this is the only possible solution to the Murasugi conditions. Suppose that $10_{162}$ did have period 3. As $\Delta_{10_{162}}(t) \equiv 1 \bmod 3$, using the mod 3 condition we see that the linking number must be $\lambda=1$. Now using the $\mathbb{Z}\left[\zeta_{3}\right]$ criterion we have that $\Delta_{10_{162}}(t)$ would factor as $\Delta_{\bar{K}}(t) F(t) F(t)^{\sigma}$, where $F(t) \in \mathbb{Z}\left[\zeta_{3}\right]\left[t, t^{-1}\right]$ and $\sigma$ is a nontrivial Galois automorphism in the Galois group of $\mathbb{Q}\left(\zeta_{3}\right)$ over $\mathbb{Q}$. However, $\Delta_{10_{162}}(t)$ is irreducible in $\mathbb{Z}\left[\zeta_{3}\right]\left[t, t^{-1}\right]$, and hence the only factor must be $\Delta_{\bar{K}}(t)=\Delta_{10_{162}}(t)$.

We now want to observe that the twisted Murasugi conditions do obstruct $10_{162}$ from having period 3 . The previous argument shows that if it does have period 3, the quotient knot $\bar{K}$ has the same polynomial. Thus, the 2 -fold branched cover of the quotient knot has homology of order $\Delta_{10_{162}}(-1)=35$. Since this is divisible by 5 , but not $5^{2}$, it follows that $\bar{K}$ has a surjective representation to the fifth dihedral group, $D_{5}$, that is unique up to composition with automorphisms of $D_{5}$. (Recall that representations to $D_{5}$ correspond to representations of the first homology of the 2-fold branched cover to $\mathbb{Z} / 5 \mathbb{Z}$.)

The group $D_{5}$ has a representation to $G L_{4}(\mathbb{Z})$. It is defined via the identification of $\mathbb{Z}^{4}$ with $\mathbb{Z}\left[\zeta_{5}\right]$, as free abelian groups: the order two automorphism is given by complex conjugation and the order 5 automorphism is given by multiplication by $\zeta_{5}$. Similar reasoning applies to the knot $10_{162}$, and so $10_{162}$ has a nontrivial representation $\rho$ to $G L_{4}(\mathbb{Z})$ which lifts a representation $\bar{\rho}$ of $\bar{K}$ with image this dihedral subgroup. 
With respect to this representation, a computation gives that

$$
\Delta_{10_{162}, \rho},(t)=\left(11 t^{8}-21 t^{6}-39 t^{4}-21 t^{2}+11\right)(t-1)^{3}(t+1)^{3} .
$$

Each factor in the above factorization is irreducible in $\mathbb{Z}\left[\zeta_{3}\right]\left[t, t^{-1}\right]$. Hence, the $\mathbb{Z}\left[\zeta_{3}\right]$ criterion in Theorem 3 implies that $\Delta_{\bar{K}, \bar{\rho}}(t)=\Delta_{10_{162}, \rho}(t)$. Given this, it becomes evident that the mod 3 condition in Theorem 4 cannot hold. Thus, the knot does not have period 3 .

This knot was already known not to have period 3: see [26] or [33]. It should, however, be noted that by a result of [9], any Alexander polynomial $\Delta$ satisfying $\Delta \equiv \pm t^{m} \bmod$ $n$ for some $m$ is the Alexander polynomial of some knot of period $n$.

\section{Alexander polynomial 1 examples}

As a second and more subtle application, we consider the two prime knots of 11 crossings having trivial Alexander polynomial: $11_{n 34}$ and $11_{n 42}$. In both cases we show that the twisted Alexander polynomial obstructs periodicity, where clearly the classical polynomial cannot. The bounds on the periods for these knots that we present here were first found by Naik [33], using geometric work of Edmonds [12], based on the theory of minimal surfaces in 3-manifolds. Alternatively, SnapPea also provides the periods of these knots. We present these examples here both to illustrate our purely algebraic approach, and to indicate the independence of the results from deep differential geometric theory.

Both of these knots have representations to the alternating group, $A_{5}$. In Figure 1 we have illustrated both knots, along with one choice of representations.

Suppose that one of these knots, we call it $K$, is periodic of period $q$ with axis $A$. Selecting a base point for the fundamental group on $A$, we have the $\mathbb{Z} / q \mathbb{Z}$ action inducing an action on the representations of $\pi_{1}\left(X_{K}\right)$ onto $A_{5}$. However, a direct calculation shows that there is a unique surjective representation up to an automorphism of $A_{5}$. (This calculation was done with Knotscape [20].) Every automorphism of $A_{5}$ is given by conjugation by an element of $S_{5}$, and in particular, the only possible orders of automorphisms are 2, 3, 4, 5, and 6 .

Let $p$ be a prime other than 2, 3, or 5 . It follows that if $K$ has period $p$, then the action fixes the representation illustrated in the figure, and thus the representation is the lift of a representation to $A_{5}$ of the quotient knot.

Algebraic 83 Geometric Topology, Volume 6 (2006) 

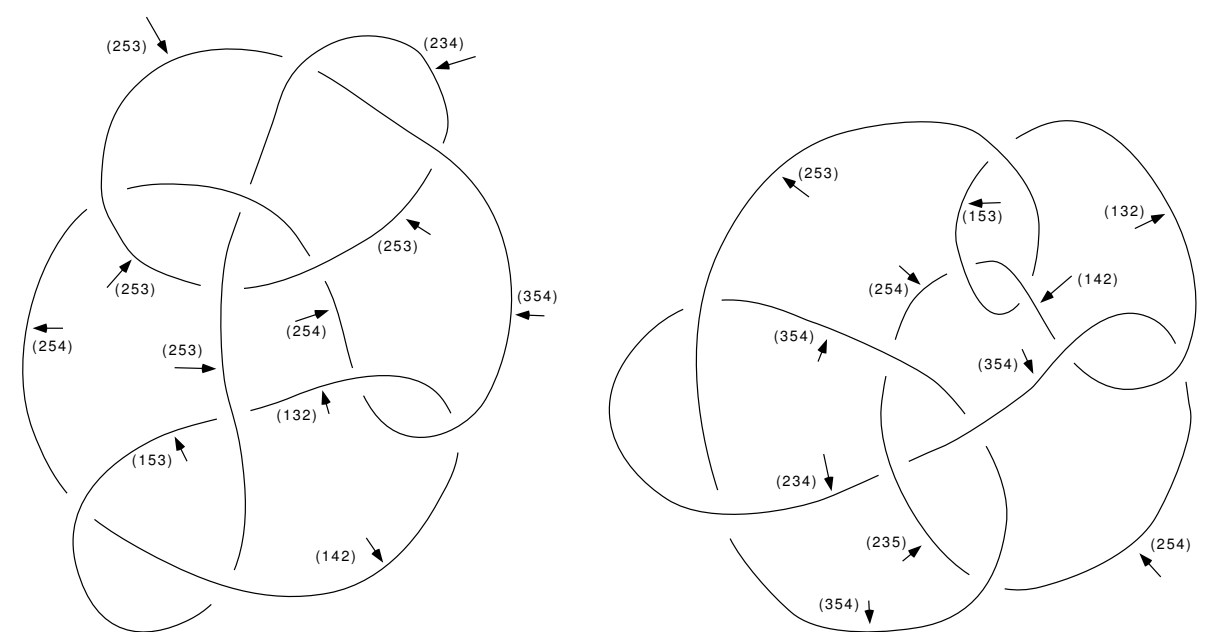

Figure 1: $11_{n 34}$ and $11_{n 42}$

\subsection{Example: 11 $1_{n 34}$}

To apply Theorems 3 and 4 we use the standard representation of $A_{5}$ acting on $\mathbb{Z}^{5}$, and then reduce to either rational or $\mathbb{Z} / p \mathbb{Z}$ coefficients. We begin with the knot $11_{n 34}$, which we abbreviate for now as $K$. Computations based on Theorem 11 and using Maple yield

$$
\Delta_{K, \rho}(t)=\left(t^{2}-t+1\right)\left(t^{2}+t+1\right)\left(5 t^{4}+t^{3}+8 t^{2}+t+5\right)(t+1)^{2}(t-1)^{4},
$$

a polynomial of degree 14 .

The exact determination of $\delta_{L, \rho}$ is not possible, but from the classical Murasugi congruence we have that $\operatorname{lk}(K, A)=\lambda=1$, and since the representation takes values in $S L_{5}(Z)$, it follows that $\delta_{L, \rho}(t)$ is a monic polynomial of degree exactly 5 .

Finally, we have by a direct computation that $\Delta_{K, \rho}^{0}=(1-t)$.

Consider the $\mathbb{Z} / p \mathbb{Z}$ criterion of Theorem 4. Since we are assuming that $p$ is not 5 , $\Delta_{K, \rho}$ is degree 14 , and so we have $4(p-1)+k p=14$, where $k$ is the degree of the $\mathbb{Z} / p \mathbb{Z}$ polynomial $\Delta_{\bar{K}, \bar{\rho}}(t)$. This gives that $(k+4) p=18$, an impossibility, given that $p$ is a prime greater than 5 .

\subsection{Example: $11_{n 42}$}

An identical calculation applies for $K_{2}=11_{n 42}$. The only change throughout is that $\Delta_{K_{2}, \rho}(t)=(t-1)^{4}\left(t^{2}+t+1\right)\left(5 t^{6}+5 t^{5}-5 t^{4}-9 t^{3}-5 t^{2}+5 t+5\right)$, a polynomial 
of degree 12. This now gives that $4(p-1)+k p=12$, or $(k+4) p=16$. Again, this is not possible for a prime greater than 5 .

\section{Additional conditions on twisted polynomials of periodic knots}

In the Sections 6 and 7 we obtained conditions on the twisted polynomials of a periodic knot when the representation of the fundamental group of the knot complement is a lift of a representation for the quotient knot. In this section we consider representations which do not factor through the fundamental group of the quotient.

Theorem 20 Suppose that the knot $K$ has period $q$. Then there is a $\mathbb{Z}_{q}$ action on the representation space $\operatorname{Hom}\left(\pi_{1}\left(S^{3}-K\right), G L_{n}(R)\right)$ and the twisted Alexander polynomials are preserved under this action.

Proof Let $f$ be the order $q$ homeomorphism of $S^{3}$ which leaves $K$ invariant and fixes a disjoint circle $A$. Choose as a base point for the knot group a point in $A$ and denote by $f_{*}$ the automorphism of the knot group induced by the restriction of $f$ to $S^{3}-K$. Let $\rho: \pi_{1}\left(S^{3}-K\right) \rightarrow G L_{n}(R)$ be a representation. The $\mathbb{Z}_{q}$ action is given by $f^{*} \rho=\rho \circ f_{*}$. Clearly, $\Delta_{K, \rho}=\Delta_{K, f^{*} \rho}$.

The usefulness of this observation is illustrated in the example below where we use homological conditions given by the untwisted Alexander polynomial to first conclude that the action on the representation space has to be nontrivial and then show that the twisted polynomials are not preserved under a nontrivial $\mathbb{Z} / q \mathbb{Z}$ action, thereby ruling out period $q$.

Example Consider the knot $K=12_{n 847}$. The Alexander polynomial for $K$ is $\Delta(t)=1-7 t+18 t^{2}-23 t^{3}+18 t^{4}-7 t^{5}+t^{6}=\left(t^{2}-t+1\right)\left(t^{2}-3 t+1\right)^{2}$. Over $\mathbb{Z}\left[\zeta_{3}\right]$ it factors as $\left(t+\zeta_{3}\right)\left(t+\zeta_{3}^{2}\right)\left(t^{2}-3 t+1\right)^{2}$ and it is congruent to $\left(1+t+t^{2}+t^{3}\right)^{2}$ mod 3. Applying the Murasugi conditions in Theorems 1 and 2 we see that, if this knot has period 3, then the polynomial of the quotient knot $\bar{K}$ has to be $\bar{\Delta}(t)=1$.

The homology of the 2-fold cover $M_{2}$ of $S^{3}$ branched over $K$ is $\mathbb{Z}_{5} \times \mathbb{Z}_{5} \times \mathbb{Z}_{3}$. The group $\mathbb{Z}_{5} \times \mathbb{Z}_{5}$ has 24 nontrivial representations to $\mathbb{Z}_{5}$. These representations correspond to 6 nontrivial conjugacy classes of representations of $\pi_{1}\left(S^{3}-K\right)$ to $D_{5}$. As in Section 8 we view these as representations to $G L_{4}(\mathbb{Z})$.

Suppose that $K$ has period 3. Again choosing a base point in the fixed set of the order 3 homeomorphism $f$ of $S^{3}$, we have a unique lift to the cover $M_{2}$ which fixes a 
chosen lift of the base point. This lift induces a $\mathbb{Z}_{3}$ action on the 5-primary subgroup $\mathbb{Z}_{5} \times \mathbb{Z}_{5}$ of $H_{1}\left(M_{2}\right)$. A transfer argument gives us that the fixed point set of this action is isomorphic to a subgroup of $H_{1}$ of the quotient $\bar{M}_{2}$, which is trivial since $\bar{\Delta}(t)=1$. (See Proposition 2.5 in [33] and Proposition 4 in [34].) It follows that the action is nontrivial.

Therefore the $\mathbb{Z}_{3}$ action on the corresponding $G L_{4}(\mathbb{Z})$ representations of the knot group is nontrivial and it permutes the 6 conjugacy classes. Therefore these representations should give at most two distinct twisted polynomials. However, explicit computations have resulted in 4 distinct twisted polynomials thus ruling out period 3 for this knot.

\section{Freely periodic knots}

Historically the main focus of the study of periodicity of knots has concerned periodic actions for which there is an axis, the "periodic knots" studied so far in this paper. It is for these knots that the periodic nature can most readily be illustrated in a knot diagram. There is a second class of periodic knots, freely periodic knots, that are invariant under a free action on the 3-sphere. These are of independent interest and also arise in the study of knots in lens spaces.

The main classical result concerning freely periodic knots is Hartley's [16]:

Theorem 21 If $K$ is a freely periodic knot of period $q$, then $\Delta_{K}\left(t^{q}\right)=\prod_{i=0}^{q-1} \bar{\Delta}\left(\zeta_{q}^{i} t\right)$ for some Alexander polynomial $\bar{\Delta}$.

In this section we will prove a generalization of Hartley's theorem to the case of twisted Alexander polynomials and illustrate the strength of the generalization by ruling out free periodicity, of all possible orders, for a 10 crossing knot that Hartley was unable to resolve. Since Hartley's theorem and its generalization call on factorizations in a cyclotomic ring, as in [16] we call on some nontrivial number theory. The number theoretic techniques we use, however, represent a great simplification over those originally used by Hartley.

In the case of free periodicity, since there is no axis, the quotient of $\left(S^{3}, K\right),(M, \bar{K})$, is a knot in a homology lens space. To simplify our considerations we will work with $R=\mathbb{Q}\left[\zeta_{q}\right]$.

Observe first that $\pi_{1}(M)=\mathbb{Z} / q \mathbb{Z}$. We next want to see that $H_{1}(M-N(\bar{K})) \cong \mathbb{Z}$. Since $M-N(\bar{K})$ has torus boundary, its homology has rank at least one. (For a 3-manifold $X$ with boundary $\partial=T^{2}$, the long exact sequence of the pair $(X, \partial)$ 
yields $\chi(\partial)-\chi(X)+\chi(X, \partial)=0$. But $\chi(\partial)=0$ and $\chi(X, \partial)=-\chi(X)$ by Poincaré duality. Thus, $\chi(X)=0$. Since $H_{3}(X)=0$ for a bounded 3-manifold, and $H_{0}=\mathbb{Z}$, it must be that the first Betti number is positive.) Attaching $N(\bar{K})$ onto $M-N(\bar{K})$ adds one relation on homology and yields $\mathbb{Z} / q \mathbb{Z}$, so $H_{1}(M-N(\bar{K}))$ must be isomorphic to either $\mathbb{Z}$ or $\mathbb{Z} \oplus \mathbb{Z} / q \mathbb{Z}$. To see that $H_{1}(M-N(\bar{K}))$ is torsion free, we recall first that $\bar{K}$ lifts to a connected curve in the $q$-fold cover of $M$. It follows that the inclusion $H_{1}(\partial N(\bar{K})) \rightarrow H_{1}(M-N(\bar{K}))$ is surjective. Thus $H_{1}(M-N(\bar{K}), \partial N(\bar{K}))=0$. By duality this implies that $H^{2}(M-N(\bar{K}))=0$. Finally the universal coefficient implies that $H_{1}(M-N(\bar{K}))$ is torsion free. In summary, we have the following lemma.

Lemma $22 H_{1}(M-\bar{K}) \cong \mathbb{Z}$ and the covering $S^{3}-K \rightarrow M-\bar{K}$ is a $q$-fold cyclic cover corresponding to a surjection of $\mathbb{Z}$ onto $\mathbb{Z} / q \mathbb{Z}$. The map $H_{1}\left(S^{3}-K\right) \rightarrow$ $H_{1}(M-\bar{K})$ is the homomorphism from $\mathbb{Z}$ to $\mathbb{Z}$ given by multiplication by $q$.

As before, we now write $X_{K}$ and $X_{\bar{K}}$ for the knot complements.

To apply our techniques to twisted polynomials of $K$, we assume the existence of a representation $\bar{\rho}: \pi_{1}\left(X_{\bar{K}}\right) \rightarrow G L_{n}(R)$. The lift of the representation to $X_{K}$ will be denoted $\rho$.

There is the natural representation $\bar{\epsilon}$ of $\pi_{1}\left(X_{\bar{K}}\right) \rightarrow \mathbb{Z}$ and thus a twisted polynomial $\Delta_{\bar{K}, \bar{\rho}}$. This lifts to give a representation $\epsilon$ of $\pi_{1}\left(X_{K}\right) \rightarrow \mathbb{Z}$, but note now that this is $q$ times the usual representation. If we denote the twisted polynomial of $K$ associated to $\rho$ and $\epsilon$ by $\Delta_{K, \rho, \epsilon}$ the next result follows immediately.

Lemma $23 \Delta_{K, \rho, \epsilon}(t)=\Delta_{K, \rho}\left(t^{q}\right)$.

We can now apply Shapiro's lemma to this situation, as was done in the proof of Theorem 3 in Section 6, to find

$$
\Delta_{K, \rho}\left(t^{q}\right)=\prod_{i=0}^{q-1} \operatorname{Ord} H_{1}\left(X_{\bar{K}}, R^{n}\left[t^{ \pm 1}\right] \otimes \mathbb{Q}\left[\zeta_{q}^{i}\right]\right) .
$$

Here the action of $\pi_{1}\left(X_{\bar{K}}\right)$ on $\mathbb{Q}\left[\zeta_{q}^{i}\right]$ is via $\epsilon$. Because of this,

$$
R^{n}\left[t^{ \pm 1}\right] \otimes \mathbb{Q}\left[\zeta_{q}^{i}\right] \cong R^{n} \otimes R\left[t^{ \pm 1}\right] \otimes \mathbb{Q}\left[\zeta_{q}^{i}\right] \cong R^{n} \otimes R\left[t^{ \pm 1}\right]
$$

(Recall that $R=\mathbb{Q}\left[\zeta_{q}\right]$.) In the last of these, the isomorphism is as abelian groups. The module structure has now changed in that the action of $\pi_{1}\left(X_{\bar{K}}\right)$ on $R\left[t^{ \pm 1}\right]$ is such that if an element in $\pi_{1}$ generates $H_{1}\left(X_{\bar{K}}\right)$, it acts by multiplication by $\zeta_{q}^{i} t$, rather than simply by $t$. 
Theorem 5 from the introduction can now be restated:

Theorem 5 In the notation above:

$$
\Delta_{K, \rho}\left(t^{q}\right)=\prod_{i=0}^{q-1} \Delta_{\bar{K}, \bar{\rho}}\left(\zeta_{q}^{i} t\right) .
$$

In the case of trivial one dimensional representations, this gives a new proof of Hartley's result, Theorem 21 .

Examples Consider the knot $10_{62}$, a knot identified by Hartley as having possible free periods that cannot be ruled out by Theorem 21 nor by the criterion in terms of homology of the 2-fold branched cover obtained in Theorem 2.2 of [16].

The Alexander polynomial for this knot is $\Delta=t^{8}-3 t^{7}+6 t^{6}-8 t^{5}+9 t^{4}-8 t^{3}+$ $6 t^{2}-3 t+1$, the product of cyclotomic polynomials, $\Phi_{10}(t)\left(\Phi_{6}(t)\right)^{2}$. As Hartley notes in [16], both the factoring condition on Alexander polynomials of freely periodic knots and the structure of $H_{1}\left(M_{2}\right)$ leave open the possibility of a free period $q$, for any $q$ which is relatively prime to 30 , and for these one would have $\Delta_{\bar{K}}=\Delta$.

Observe that $\Delta_{\bar{K}}(-1)=45$. Thus, the quotient knot complement would have a $\mathbb{Z}_{5}$ in the homology of the 2 -fold cyclic branched cover giving a nontrivial representation of the knot group onto the dihedral group $D_{5}$ which would lift to a nontrivial representation of the group of $10_{62}$ onto $D_{5}$. A computer calculation of the associated twisted polynomial yields a degree 28 polynomial with a degree 8 irreducible factor $f(t)=$ $t^{8}-3 t^{6}+3 t^{4}-3 t^{2}+1$ with multiplicity 2 .

If $10_{62}$ has free period $q$, then applying Theorem 5 we would have

$$
f\left(t^{q}\right)=g(t) \prod_{i=1}^{q-1} g\left(\zeta_{q}^{i} t\right),
$$

for some rational polynomial $g$. (Here we have used that $\mathbb{Q}\left[\zeta_{q}\right][t]$ is a UFD to conclude that the factorization given in Theorem 5 determines a similar factorization for each factor which is irreducible over $\mathbb{Q}$.) We want to show that such a factorization cannot exist. As we are showing that there are no free periods, it is sufficient to rule out periods of prime order, so we assume henceforth that $q$ is prime. For $q \leq 11$ that $f\left(t^{q}\right)$ has no such factorization can be checked via computer, so we assume that $q \geq 13$.

Initially we know only that $g$ is a rational polynomial and that the equality is true only up to a rational multiple; we now want to observe that $g$ can be assumed to be integral 
and that the equality is exact. If we replace $g$ with its monic associate we see the equality becomes necessarily exact. Note that evaluating the equation above at $t=0$ yields that $g$ has constant term 1 .

We now want to show that $g$ has integer coefficients. Each root of $g$ is the root of some irreducible factor of $g$, which itself must be a factor of $f$. But the monic irreducible factors of a monic integral polynomial are integral. Thus the product of the monic irreducible factors of $g$ is also integral, and so $g$ is as well.

There is a homomorphism $\mathbb{Z}\left[\zeta_{q}\right] \rightarrow \mathbb{Z} / q \mathbb{Z}$ sending $\zeta_{q}$ to 1 . Applying this to the polynomial factorization and recalling that $f\left(t^{q}\right) \equiv(f(t))^{q} \bmod q$, we have

$$
f(t)^{q} \equiv(g(t))^{q} \bmod q .
$$

From this it follows that

$$
f(t)=g(t)+q h(t)
$$

for some integral polynomial $h$. Since $f$ and $g$ are monic of degree 8, we know that $h$ is of degree 7 or less. We also know that $1=f(0)=g(0)+q h(0)$ and $g(0)=1$, so $h(0)=0$.

Next observe that $g(1)$ divides $f(1)=-1$, so $g(1)= \pm 1$. Since $f(1)=g(1)+q h(1)$, we have that $1- \pm 1=q h(1)$, so clearly $h(1)=0$ and $g(1)=-1$.

Similarly, $g(-1)$ divides $f(-1)=-1$, so $g(-1)= \pm 1$. But $f(-1)=g(-1)+$ $q h(-1)$, so again $h(-1)=0$ and $g(-1)=-1$.

Next we move to the ring of Gaussian integers, $\mathbb{Z}[i]$, and observe that $g(i)$ divides $f(i)=11$. It is easily checked that 11 is prime in $\mathbb{Z}[i]$, so we have that $g(i)=$ $\pm 1, \pm i, \pm 11$, or $\pm 11 i$. In none of these cases would $f(i)-g(i)$ be divisible by $q$ in $\mathbb{Z}[i]$ (we are assuming that $q \geq 13$ ). Thus $h(i)=0$.

By conjugation we have $h(-i)=0$.

Now we move to the ring $\mathbb{Z}\left[\zeta_{3}\right]$. A computation shows that $f\left(\zeta_{3}\right)=5 \zeta_{3}$. The only units in $\mathbb{Z}\left[\zeta_{3}\right]$ are of the form $\pm \zeta_{3}^{i}$, and we will let $u$ denote a unit of this form. Then $g\left(\zeta_{3}\right)=u$ or $g\left(\zeta_{3}\right)=5 u$. One now checks that for none of the possibilities is $f\left(\zeta_{3}\right)-g\left(\zeta_{3}\right)$ divisible by $q$ (mostly simply, none of the norms of each is divisible by the square of a prime greater than 11). Hence, $h\left(\zeta_{3}\right)=0$. Conjugating we have also $h\left(\zeta_{3}^{2}\right)=0$.

Finally we work in $\mathbb{Z}\left[\zeta_{6}\right]=\mathbb{Z}\left[\zeta_{3}\right]\left(\zeta_{6}=-\zeta_{3}\right)$. Now we find that $f\left(\zeta_{6}\right)=-5 \zeta_{6}=5 \zeta_{3}$. As in the previous paragraph it follows that $h\left(\zeta_{6}\right)=h\left(\zeta_{6}^{5}\right)=0$.

At this point we have found nine zeros for the degree 7 polynomial $h$, and thus $h$ is identically 0 . We have now that $g(t)=f(t)$, so that $f(t)$ is a factor of $f\left(t^{q}\right)$. But 
$f$ has eight distinct roots and the one with largest norm, say $\alpha$, is real, with value approximately 1.47. This leads to a contradiction: since $f(t)$ divides $f\left(t^{q}\right)$, we have that $f\left(\alpha^{q}\right)=0$. But $\alpha^{q}$ has norm greater than any roots of $f$.

Thus, we have proved that $10_{62}$ does not have any free periods.

Note that all free periods for knots up to 10-crossings not settled by Hartley were determined by the combined works of Boileau and Zimmermann in [2] and Sakuma in [37]. In particular, it was shown in [2] that $10_{62}$ is not freely periodic. The proofs in [2] and [37] strongly rely on the hyperbolic structure on the complement unlike our proof above which is based only on homological algebra.

Also note that Chbili in [7] obtains criteria for free periodicity in terms of the HOMFLYPT polynomial. These criteria are then applied to examples in [16] for which free periods could not be eliminated using Theorem 21 alone. Free periods for $K=10_{62}$ are not ruled out in [7].

\section{References}

[1] C Adams, M Hildebrand, J Weeks, Hyperbolic invariants of knots and links, Trans. Amer. Math. Soc. 326 (1991) 1-56 MR994161

[2] M Boileau, B Zimmermann, Symmetries of nonelliptic Montesinos links, Math. Ann. 277 (1987) 563-584 MR891592

[3] KS Brown, Cohomology of groups, Graduate Texts in Mathematics 87, Springer, New York (1994) MR1324339

[4] G Burde, Über periodische Knoten, Arch. Math. (Basel) 30 (1978) 487-492 MR0645216

[5] G Burde, H Zieschang, Knots, de Gruyter Studies in Mathematics 5, Walter de Gruyter \& Co., Berlin (2003) MR1959408

[6] J C Cha, Fibred knots and twisted Alexander invariants, Trans. Amer. Math. Soc. 355 (2003) 4187-4200 MR1990582

[7] N Chbili, The skein polynomial of freely periodic knots, Topology Appl. 121 (2002) 535-542 MR1909007

[8] J I Cogolludo, V Florens, Twisted Alexander polynomials of plane algebraic curves arXiv:math.GT/0504356

[9] J F Davis, C Livingston, Alexander polynomials of periodic knots, Topology 30 (1991) 551-564 MR1133872

[10] J F Davis, C Livingston, Periodic knots, Smith theory, and Murasugi's congruence, Enseign. Math. (2) 37 (1991) 1-9 MR1115739 
[11] C H Dowker, M B Thistlethwaite, Classification of knot projections, Topology Appl. 16 (1983) 19-31 MR702617

[12] A L Edmonds, Least area Seifert surfaces and periodic knots, Topology Appl. 18 (1984) 109-113 MR769284

[13] S Friedl, T Kim, The Thurston norm, fibered manifolds and twisted Alexander polynomials arXiv:math.GT/0505594

[14] H Goda, T Kitano, T Morifuji, Reidemeister torsion, twisted Alexander polynomial and fibered knots, Comment. Math. Helv. 80 (2005) 51-61 MR2130565

[15] H Goda, T Morifuji, Twisted Alexander polynomial for SL(2, C) -representations and fibered knots, C. R. Math. Acad. Sci. Soc. R. Can. 25 (2003) 97-101 MR2013157

[16] R Hartley, Knots with free period, Canad. J. Math. 33 (1981) 91-102 MR608857

[17] J A Hillman, New proofs of two theorems on periodic knots, Arch. Math. (Basel) 37 (1981) 457-461 MR643289

[18] J A Hillman, On the Alexander polynomial of a cyclically periodic knot, Proc. Amer. Math. Soc. 89 (1983) 155-156 MR706531

[19] J Hillman, Algebraic invariants of links, Series on Knots and Everything 32, World Scientific Publishing Co., River Edge, NJ (2002) MR1932169

[20] J Hoste, M Thistlethwaite, Knotscape, computer program available at www . math.utk. edu/ morwen

[21] B J Jiang, S C Wang, Twisted topological invariants associated with representations, from: "Topics in knot theory (Erzurum, 1992)", NATO Adv. Sci. Inst. Ser. C Math. Phys. Sci. 399, Kluwer Acad. Publ., Dordrecht (1993) 211-227 MR1257911

[22] P Kirk, C Livingston, Twisted Alexander invariants, Reidemeister torsion, and CassonGordon invariants, Topology 38 (1999) 635-661 MR1670420

[23] P Kirk, C Livingston, Twisted knot polynomials: inversion, mutation and concordance, Topology 38 (1999) 663-671 MR1670424

[24] T Kitano, Twisted Alexander polynomial and Reidemeister torsion, Pacific J. Math. 174 (1996) 431-442 MR1405595

[25] T Kitano, M Suzuki, A partial order in the knot table, University of Tokyo Graduate School of Mathematical Sciences, UTMS 2005-7 (March 8, 2005)

[26] K Kodama, M Sakuma, Symmetry groups of prime knots up to 10 crossings, from: “Knots 90 (Osaka, 1990)”, de Gruyter, Berlin (1992) 323-340 MR1177431

[27] W P Li, L Xu, Counting $\mathrm{SL}_{2}\left(\mathbf{F}_{2}\right.$ s) representations of torus knot groups, Acta Math. Sin. (Engl. Ser.) 19 (2003) 233-244 MR1987793

[28] X S Lin, Representations of knot groups and twisted Alexander polynomials, Acta Math. Sin. (Engl. Ser.) 17 (2001) 361-380 MR1852950 
[29] C Livingston, J C Cha, KnotInfo: Table of Knots, www . indiana. edu/ knotinfo

[30] T Morifuji, A Torres condition for twisted Alexander polynomials arXiv: math.GT/0412434

[31] T Morifuji, A twisted invariant for finitely presentable groups, Proc. Japan Acad. Ser. A Math. Sci. 76 (2000) 143-145 MR1801675

[32] K Murasugi, On periodic knots, Comment. Math. Helv. 46 (1971) 162-174 MR0292060

[33] S Naik, Periodicity, genera and Alexander polynomials of knots, Pacific J. Math. 166 (1994) 357-371 MR1313460

[34] S Naik, New invariants of periodic knots, Math. Proc. Cambridge Philos. Soc. 122 (1997) 281-290 MR1458233

[35] J H Przytycki, On Murasugi's and Traczyk's criteria for periodic links, Math. Ann. 283 (1989) 465-478 MR985242

[36] M Sakuma, On the polynomials of periodic links, Math. Ann. 257 (1981) 487-494 MR639581

[37] M Sakuma, Non-free-periodicity of amphicheiral hyperbolic knots, from: "Homotopy theory and related topics (Kyoto, 1984)", Adv. Stud. Pure Math. 9, North-Holland, Amsterdam (1987) 189-194 MR896954

[38] A Tamulis, Knots of ten or fewer crossings of algebraic order 2, J. Knot Theory Ramifications 11 (2002) 211-222 MR1895371

[39] P Traczyk, $10_{101}$ has no period 7: a criterion for periodic links, Proc. Amer. Math. Soc. 108 (1990) 845-846 MR1031676

[40] H F Trotter, Periodic automorphisms of groups and knots, Duke Math. J. 28 (1961) 553-557 MR0133820

[41] M Wada, Twisted Alexander polynomial for finitely presentable groups, Topology 33 (1994) 241-256 MR1273784

[42] J Weeks, SnapPea, available at www .geometrygames .org/

[43] Y Yokota, The skein polynomial of periodic knots, Math. Ann. 291 (1991) 281-291 MR1129368

School of Mathematics and Statistics F07, University of Sydney

NSW 2006, Australia

Department of Mathematics, Indiana University

Bloomington IN 47405, USA

Department of Mathematics and Statistics, University of Nevada

Reno NV 89557, USA

jonh@maths.usyd.edu.au, livingst@indiana.edu, naik@unr.edu

Received: 17 June 2005 Revised: 24 January 2006

Algebraic $\mathcal{E}$ Geometric $\mathcal{T}$ opology, Volume 6 (2006) 